\title{
ACOLHIMENTO FAMILIAR COLETIVO E INDIVIDUAL EM ALIMENTAÇÃO E NUTRIÇÃO
}

Originais recebidos em: 1\%12/2010

Aceito para publicação em: 17/08/2011

Monique Vicente Rocha Janaina das Neves Anelise Regina Royer Pinto Neila Maria Viçosa Machado Patrícia Maria de Oliveira Natali Carol Fritzen Universidade Federal de Santa Catarina nats.carol@gmail.com

\section{Resumo}

O Acolhimento Familiar Coletivo e Individual em Alimentação e Nutrição foi uma ação do Projeto Creches Saudáveis que teve por objetivo realizar acompanhamento de informações relacionadas à saúde infantil no espaço escolar para grupos de crianças prioritários, buscando promover a saúde e conhecer a rede social e a cultura alimentar dessas crianças. O Projeto teve duração de 24 meses e foi realizado em sete Unidades de Educação Infantil da Secretaria Municipal de Educação de Florianópolis/SC. Ao total foram realizadas três avaliações antropométricas, além de coletas de informações. No segundo ano do projeto, entre os meses de abril e outubro do ano de 2009 , as famílias das crianças que permaneceram com o diagnóstico nutricional abaixo ou acima do esperado em qualquer um dos três índices antropométricos (peso para idade, estatura para idade e peso para estatura) foram convidadas a participar de encontros mensais com os participantes do projeto, em grupo e individualmente. Foi observada uma tendência ao peso elevado.

Palavras-chave: Projeto Creches Saudáveis. Acolhimento Familiar. Educação Infantil. Promoção da Saúde. Direito Humano - Alimentação Adequada.

COLLECTIVE AND INDIVIDUAL FAMILY RECEPTION IN FOOD AND NUTRITION

\begin{abstract}
The "Collective and Individual Family Reception in Food and Nutrition" is an action of "Project Healthy Day Care Centers" aimed to monitor important information on child health for priority groups of children at day care centers, seeking to promote health and to know the social network and food culture of these children. After measuring height and weight, the families of children who had inappropriate nutritional diagnosis for any of the three anthropometric indexes (weight for age, height for age and weight for height) were invited to participate in monthly meetings with project participants, either in groups or individually. A tendency to high weight was observed among these children. The proposal was carried out between April and October of 2009.
\end{abstract}

Keywords: Project Healthy Day Care Centers; Family Reception; Child Education; Health Promotion; Human Right - Adequate Food 


\section{INTRODUÇÃO}

Em 1948, a Assembleia Geral da Organização das Nações Unidas proclamou a Declaração Universal dos Direitos Humanos (ONU, 1948), que garante a todos o direito à liberdade (de pensamento, de religião, de expressão e locomoção), à família, ao emprego e remuneração justa, ao acesso à saúde, à moradia, à alimentação e à educação.

Dentro dos princípios dessa Declaração, surge o Direito Humano à Alimentação Adequada (DHAA), que tem como dimensões indivisíveis o direito de estar livre da fome e da má nutrição e o direito à alimentação adequada (BURITY et al., 2010). A opção por trabalhar com o DHAA no Projeto Creches Saudáveis pressupôs a relação entre educação e saúde a partir do acesso à alimentação e nutrição adequadas por meio de ações de educação permanente com professores; de acolhimento em alimentação e nutrição com famílias; e de efetivação de um sistema de vigilância alimentar e nutricional, na perspectiva do controle social.

A concepção da Educação Popular em Saúde (BRASIL, 2007) foi utilizada para desenvolver ações relacionadas ao DHAA e despertar nas pessoas a percepção de protagonistas das melhorias na sua comunidade, adquirindo responsabilidade pela sua própria saúde e da coletividade. Além disso, buscou-se também criar um elo entre as necessidades e expectativas das pessoas e o Centro de Saúde da Família, estabelecendo um fluxo contínuo de informações entre a saúde e a educação.

Entre as ações destacadas acima, o Acolhimento em Alimentação e Nutrição, enfoque deste artigo, foi baseado nos princípios do HumanizaSUS (BRASIL, 2010), instituído pelo Ministério da Saúde em 2003 com o objetivo de garantir que o Sistema Único de Saúde atenda igualmente às necessidades de todos de uma maneira acolhedora e humanitária.

A realização do Acolhimento em Alimentação e Nutrição envolve questões intersetoriais e interdisciplinares, a partir do momento que cria um elo entre a unidade educativa e a unidade de saúde, proporcionando uma articulação entre os diversos profissionais envolvidos. Além disso, o Acolhimento em Alimentação e Nutrição é uma proposta inovadora e, portanto, com poucas publicações encontradas na literatura, que trata de um tema relevante para a saúde das crianças, tendo em vista a importância da participação dos pais, da escola e da unidade de saúde na promoção da saúde de crianças em idade pré-escolar.

O Acolhimento Familiar e Individual em Alimentação e Nutrição teve por objetivo realizar acompanhamento de informações importantes para a saúde infantil no espaço escolar para grupos de crianças prioritários, visando potencializar o saudável e garantir a promoção à 
saúde, além de conhecer a rede social e a cultura alimentar da criança e apoderar as famílias sobre situações de saúde que podem ser melhoradas.

\section{MATERIAL E MÉTODOS}

O projeto foi desenvolvido em sete Unidades Educativas: Creche Fermínio Francisco Vieira, Creche Irmão Celso, Creche Vila Cachoeira, Creche Diamantina Bertolina da Conceição, Núcleo de Educação Infantil Nei Barreira do Janga, Conselho Comunitário do Monte Verde, e Filantropia Hildo Luíz de Souza, todas localizadas no município de Florianópolis. Durante a execução do Projeto, foram assistidas 751 crianças de zero a sete anos. O projeto estava inserido no Centro Colaborador de Alimentação e Nutrição do Escolar (CECANE/SC), vinculado à Universidade Federal de Santa Catarina (UFSC).

Inicialmente foi realizado o processo de territorialização, que consiste no levantamento de dados sobre as características do espaço físico do bairro, como calçadas, saneamento básico, presença de pavimentos, entre outros; dados da comunidade, como número de habitantes e de crianças; dados de trabalho e renda dos habitantes; e dados da creche, como espaço de lazer e salas de aula. $\mathrm{O}$ reconhecimento foi realizado nas proximidades da Unidade de Ensino Infantil (UEI), onde vivia a maior parte das famílias das crianças matriculadas.

A etapa seguinte consistiu na segunda antropometria, já que uma havia sido realizada no ano anterior. Esse processo, que envolve a coleta do peso e estatura das crianças, foi realizado de acordo com a metodologia proposta por LOHMAN; ROCHE; MARTORELL, (1991).

As crianças menores de dois anos foram pesadas e medidas sem roupas, fraldas e sem qualquer tipo de adereço para que a medição antropométrica fosse precisa. Para isso, foi utilizada uma balança digital Marte com capacidade máxima de $15 \mathrm{~kg}$ e divisões de $5 \mathrm{~g}$. A mensuração do comprimento foi realizada com antropômetro horizontal de madeira em posição de decúbito dorsal. Para a medição das crianças maiores de dois anos, o peso corporal foi realizado sem calçados (sapatos, tênis ou sandálias) e com roupas leves, em uma balança com capacidade de $200 \mathrm{~kg}$ e precisão de $50 \mathrm{~g}$. A estatura foi mensurada no momento da inspiração em crianças descalças e com cabelos soltos, com calcanhares unidos, braços relaxados ao longo do corpo e com a cabeça posicionada no plano horizontal de Frankfurt. Para isso, foi utilizado um estadiômetro da marca Alturaexata com 2 metros e precisão em 
milímetros. Para posterior análise e acompanhamento do crescimento e desenvolvimento individual e do grupo avaliado, foram realizadas mensurações a cada seis meses.

$\mathrm{Na}$ realização da antropometria, os bolsistas passaram por uma capacitação com o objetivo de aprender a manipular os seguintes instrumentos: balança pediátrica, balança para adultos, estadiômetro e antropômetro horizontal. Foram orientados também a utilizar uma metodologia de sensibilização, a fim de familiarizarem as crianças com os instrumentos e inserir o professor na atividade.

A cada avaliação nutricional, a antropometria foi realizada em dois períodos diferentes em cada creche, além de um período extra para pesar e medir as crianças que estavam ausentes nas duas medições anteriores, para que assim fosse possível abranger o número máximo de crianças das unidades educativas.

Os dados obtidos foram anotados manualmente em planilhas e depois foram passados para o banco de dados das creches. A análise dos dados antropométricos foi realizada seguindo a recomendação do Ministério da Saúde apresentada nos dois sítios de consulta: Sistema Nacional de Vigilância Alimentar e Nutricional (SISVAN) e Coordenação Geral da Política Nacional de Alimentação e Nutrição (CGPAN), nos quais foram encontradas as curvas de crescimento da Organização Mundial de Saúde (OMS) para crianças de zero a cinco anos (WHO, 2006) e para jovens de cinco a dezenove anos (WHO, 2007); a caderneta da criança; gráficos e tabelas das curvas da OMS em português; e a classificação do estado nutricional. Foi utilizado também o programa Anthro da OMS como uma ferramenta para a inserção dos dados coletados e classificação do estado nutricional.

Todos os dados coletados retornaram à unidade educativa para possibilitar à diretora $\mathrm{e}$ aos professores a transmissão de informações aos pais ou responsáveis sobre o estado nutricional das crianças. Dessa forma, foi entregue à direção uma lista com os dados antropométricos e avaliações nutricionais das crianças matriculadas. Além disso, foi utilizado um instrumento para representar a tendência de variação do estado nutricional da escola como um todo, o Histograma (CECANE/SC, 2009). Foram confeccionados dois histogramas, um deles relativo ao índice de peso para idade e outro à altura pela idade.

Outro instrumento utilizado foi o Quadro de Tendências (CECANE/SC, 2009), que reflete a disposição do estado nutricional de cada criança de uma mesma sala de aula, tendo a vantagem de poder avaliar a evolução do seu crescimento periodicamente.

Os Quadros de Tendências foram confeccionados a partir dos dados encontrados e entregues a cada sala das creches envolvidas no projeto; e, para que os professores ficassem 
aptos a repassar a informação aos pais, foi explicada a eles a metodologia dos Quadros de Tendência.

Para os casos de peso adequado ou baixo peso, a linha ascendente demonstra um bom resultado, já a linha reta sugere atenção e a linha em declínio implica a necessidade de encaminhar a criança para o Centro de Saúde da Família. Nos casos de peso elevado, a linha ascendente é preocupante, pois reflete ganho de peso, nesse caso, também se recomenda o encaminhamento da criança para o Centro de Saúde da Família; a linha reta é satisfatória, pois demonstra que a criança está com peso estável; sendo desejável a linha descendente, pois evidencia perda de peso. Ao utilizar esses três exemplos de retorno de resultado para as UEIs, é possível perceber que a avaliação nutricional pode ir além do entendimento de que eles estão relacionados com a doença, possibilitando avanços em direção à promoção do saudável no espaço escolar. (CECANE/SC, 2009).

A partir dos dados obtidos na antropometria, foi confeccionado pelo projeto o diagnóstico de saúde, cujos dados foram coletados nos centros de saúde, e, com equipes de saúde da família, obtiveram-se as informações referentes aos cuidados com a saúde e o processo de adoecer das crianças, com base em pesquisa nos prontuários em formatos impressos e eletrônicos do INFOSaúde. Esse diagnóstico foi aplicado com as crianças que se encontravam com baixo peso, peso elevado e/ou baixa estatura na última avaliação.

As crianças que apresentaram esses diagnósticos foram encaminhadas ao Centro de Saúde por meio do Contagem do Potencial de Saúde, instrumento criado pelo Projeto Creches Saudáveis que leva em consideração o Diagnóstico de Saúde e a análise da territorialização, atribuindo pontuações para cada aspecto.

Foi dada atenção especial para as crianças que se encontravam nessas faixas por duas antropometrias consecutivas. Com as famílias dessas crianças foi realizado, no espaço da creche, o Acolhimento Familiar Coletivo e Individual. Os encontros coletivos mensais foram embasados nos "Dez Passos para uma Alimentação Saudável - Guia Alimentar para Crianças menores de 2 anos" (BRASIL, 2002), e no "Guia Alimentar para a População Brasileira Promovendo a Alimentação Saudável” (BRASIL, 2006), para maiores de dois anos. Foram trabalhados, em média, dois passos ou diretrizes por encontro.

O Acolhimento Familiar Individual foi realizado entre as nutricionistas do Projeto Creches Saudáveis e cada família do encontro coletivo separadamente. O Projeto optou por iniciar o Acolhimento Familiar Individual após dois meses do início do Acolhimento Familiar Coletivo. Esse período serviu para fortalecer o vínculo entre a família e o profissional, 
propiciando o desvelar da estrutura familiar e suas relações (entre si, com a sociedade e instituições), eventos relevantes, procedência, hábitos alimentares e outras questões que vieram a surgir.

Nos encontros individuais foram construídos, com cada uma das famílias, o ecomapa e o genograma (CECANE/SC, 2009). O ecomapa é um instrumento utilizado pelo Serviço Social e Psicologia, adaptado à nutrição pelos residentes do Programa de Residência Interdisciplinar em Saúde da Família da Universidade Federal de Santa Catarina que desenvolveram estágio específico junto ao Projeto, esse instrumento possibilita conhecer a rede social e a cultura alimentar da comunidade. O genograma, por sua vez, trata do vínculo entre a família e o profisssional, propiciando o desvelar da estrutura familiar e suas relações (entre si, com a sociedade e instituições), eventos relevantes, procedência, hábitos alimentares e outras questões que surgirem. Primeiramente foi aplicado o genograma em várias reuniões com duração de uma hora, traçando, assim, perfil da família em questão. Em seguida fez-se o ecomapa das famílias com o objetivo de visualizar a inserção na comunidade onde vive e sua atuação como atores sociais.

\section{RESULTADOS E ANÁLISE}

$\mathrm{Na}$ antropometria realizada no primeiro semestre de 2009, foram avaliadas 751 crianças, destas 427 eram pré-escolares novos e, portanto, não participaram da antropometria do ano anterior, não atendendo aos critérios necessários para participar do acolhimento. $\mathrm{O}$ total de faltantes foi 44 pré-escolares. Os resultados encontrados nas coletas de peso (Tabela 1) e altura (Tabela 2) dos pré-escolares são apresentados à continuação.

Tabela 1: Frequência de diagnóstico nutricional observado em crianças avaliadas pelo Projeto Creches saudáveis em 2009, de acordo com o índice peso para idade.

\begin{tabular}{l|c|c|c}
\hline \multicolumn{1}{c|}{ Diagnóstico } & $\begin{array}{c}\text { Número de } \\
\text { crianças }\end{array}$ & $\begin{array}{c}\text { Frequência } \\
\text { esperada } \\
\text { (\%) }\end{array}$ & $\begin{array}{c}\text { Frequência } \\
\text { observada } \\
\text { (\%) }\end{array}$ \\
\hline Total de crianças com baixo peso & 12 & 3 & 1,60 \\
Total de crianças em monitoramento para baixo peso & 84 & 12 & 11,10 \\
Total de crianças com peso adequado & 510 & 70 & 68 \\
Total de crianças em monitoramento peso elevado & 105 & 12 & 14 \\
Total de crianças com peso elevado & 40 & 3 & 5,30 \\
Total de crianças avaliadas na Creche & $\mathbf{7 5 1}$ & $\mathbf{1 0 0}$ & $\mathbf{1 0 0}$ \\
\hline
\end{tabular}

Fonte: elaborado pelos autores. 
Os resultados encontrados no diagnostico nutricional pelo índice peso para idade, na Tabela 1, revelam que as crianças apresentam tendência ao monitoramento para peso elevado e peso elevado, sendo a frequência observada de peso elevado quase duas vezes maior que a esperada em uma população saudável.

Ao assumirmos que a distribuição da população é normal, espera-se que, em uma população saudável, seja encontrado até 3\% para baixo peso ou peso elevado, aproximadamente $12 \%$ nas faixas de monitoramento, e por volta de $70 \%$ para peso adequado. Com base nos resultados obtidos nos histogramas, pode-se notar que as faixas de baixo peso e monitoramento para baixo peso encontram-se dentro do limite estabelecido pela OMS, a faixa de peso adequado está próxima do recomendado, mas as faixas de peso elevado e monitoramento para peso elevado estão acima dos limites.

Tabela 2: Histograma comparativo de altura para idade.

\begin{tabular}{l|c|c|c}
\hline \multicolumn{1}{c|}{ Diagnóstico } & $\begin{array}{c}\text { Número de } \\
\text { crianças }\end{array}$ & $\begin{array}{c}\text { Frequência } \\
\text { esperada } \\
(\%)\end{array}$ & $\begin{array}{c}\text { Frequência } \\
\text { observada } \\
\text { (\%) }\end{array}$ \\
\hline Total de crianças com baixa estatura & 30 & 3 & 4 \\
Total de crianças com estatura adequada & 721 & 97 & 96 \\
Total de crianças avaliadas na Creche & $\mathbf{7 5 1}$ & $\mathbf{1 0 0}$ & $\mathbf{1 0 0}$ \\
\hline
\end{tabular}

Fonte: elaborado pelos autores.

Os resultados encontrados no diagnostico nutricional pelo índice de estatura para idade, na Tabela 2, informam que as crianças apresentam estatura adequada, embora a frequência de baixa estatura observada esteja maior do que a esperada para uma população saudável.

Com o decorrer do Projeto Creches Saudáveis, pôde-se perceber que foi importante realizar uma análise individual e coletiva dos dados para possibilitar ao professor o conhecimento da tendência do estado nutricional de seu grupo e para que, a partir desse resultado, ele esteja apto a adaptar a pedagogização do alimento e/ou da alimentação escolar em sala de aula à realidade; possibilita, ainda, conhecer cada criança. Sua importância se mostra também no fato de que o conhecimento do resultado individual é capaz de desmistificar sobre as necessidades da alimentação e nutrição, revelando que alimentação escolar não é a única responsável pela realidade nutricional da criança, mesmo com a presença de vulnerabilidade socioeconômica. 
Além disso, a investigação individual, levando em consideração informações coletadas sobre a comunidade e o diagnóstico de saúde e do espaço escolar, fornece mais subsídios para tomada de decisão em saúde, visto que, ao analisar os dados conjuntamente, pode-se definir se o crescimento e desenvolvimento da criança podem ser considerados saudáveis ou se ela precisa de cuidados especiais.

O Projeto Creches Saudáveis criou o Contagem do Potencial de Saúde como instrumento de promoção à saúde e controle social e para manter o sistema de monitoramento de algumas informações relevantes para a saúde infantil dos grupos prioritários acompanhados. Seu objetivo é dar ênfase às características que potencializam o saudável, e não a doença, garantindo a promoção da saúde em todos os seus aspectos. Nos casos de crianças que não estavam com o peso ou a estatura adequados, esse instrumento foi discutido com profissionais de saúde, em reuniões de área das equipes de saúde da família, a fim de subsidiar o encaminhamento necessário.

O Acolhimento Familiar Coletivo, realizado em grupo com as famílias, aconteceu nos meses de junho, julho e agosto de 2009. O Acolhimento Individual, realizado entre cada família e as nutricionistas do Projeto Creches Saudáveis, aconteceu na sequência, nos meses de agosto, setembro e outubro de 2009.

Entre todas as crianças das creches, 29 se encontravam nas faixas de risco por duas antropometrias consecutivas, e por isso suas famílias foram encaminhadas ao acolhimento. Desse total, 11 compareceram em pelo menos dois encontros coletivos e, portanto, deram continuidade à proposta, passando a participar dos encontros individuais. No entanto, duas famílias não compareceram a esses encontros.

Conforme apresentado na Tabela 3, três Unidades de Ensino Infantil realizaram reuniões com todas as famílias das crianças que entraram para o Acolhimento, já as demais não alcançaram essa frequência. As sete creches concluíram o primeiro encontro de acolhimento coletivo com os pais; no entanto, apenas quatro concluíram os outros dois encontros. Nas outras creches, os pais não compareceram ou não quiseram participar. O quarto e o quinto encontros coletivos não foram aplicados pelo projeto devido à dificuldade em reunir as famílias em um mesmo horário e, portanto, houve mudanças no planejamento. Quatro creches realizaram os dois primeiros encontros individuais. 
Tabela 3: Frequência de realização de encontros e de comparecimento de famílias no Acolhimento Coletivo e Individual.

\begin{tabular}{l|l|lccc}
\hline \multicolumn{2}{c}{ Acolhimento } & $\begin{array}{l}N^{\circ} \text { de creches que } \\
\text { realizaram }\end{array}$ & $\begin{array}{l}N^{\circ} \text { de famílias } \\
\text { compareceram }\end{array}$ & que \\
\hline \multirow{2}{*}{ Coletivo } & $1^{\circ}$ encontro & 7 & 16 \\
& $2^{\circ}$ encontro & 4 & 11 \\
& $3^{\circ}$ encontro & 4 & 7 \\
\hline Individual & $1^{\circ}$ encontro & 4 & 9 \\
& $2^{\circ}$ encontro & 4 & 6 \\
\hline
\end{tabular}

Fonte: elaborado pelos autores.

Os principais motivos levantados pelo Projeto Creches Saudáveis para o índice de faltas registrado foram a dificuldade em conciliar os horários das famílias, o pouco tempo disponível dos pais e o receio de estarem expostos em ambientes de risco para o vírus H1N1, já que os encontros aconteceram em época de surto da doença.

Tanto durante o acolhimento individual como durante o coletivo, foi percebida a dificuldade de os pais entenderem a proposta de educação do Projeto Creches Saudáveis. Foi constatado que esperavam uma medida pontual de saúde e não as inter-relações que os instrumentos ecomapa e genograma tinham como objetivo. Pelo fato de não terem a mesma visão do projeto, os pais não tinham estímulo para participar dos encontros, o que justifica também o número significativo de faltas. Diante disso, percebe-se a necessidade de desenvolver as ações em um período de tempo maior para apoderar os pais sobre as questões relacionadas à saúde, em seu conceito ampliado, para tirar a expectativa de que eles receberiam um esquema alimentar com indicação de alimentos que deveriam ingerir ou evitar.

O Projeto optou por realizar ambos os acolhimentos pela mesma nutricionista responsável, para que as famílias ficassem mais à vontade durante o acolhimento individual, visto que já teriam criado um vínculo com o profissional nos encontros coletivos.

Foi percebido também que deveriam ter acontecido mais encontros coletivos e individuais, a fim de trabalhar com os familiares todas as diretrizes do Guia Alimentar para a População Brasileira, e também questões relacionadas ao conceito ampliado de saúde.

Durante os encontros individuais, as famílias foram incentivadas a procurar o Centro de Saúde da Família, criando, assim, uma porta de entrada para o SUS, para que elas tivessem uma assistência integral em saúde. $\mathrm{O}$ objetivo desse incentivo foi criar um vínculo entre as famílias e a unidade de saúde, para que elas tivessem autonomia em procurá-lo sem que o projeto precisasse intervir. 
De acordo com o andamento do Projeto, foi perceptível a importância da realização da territorialização, tendo em vista que o conhecimento do território onde a UEI está localizada é essencial para efetivar a intersetorialidade com instituições presentes que possam constituir uma relação com a educação. Além disso, é considerado também que o conhecimento da comunidade onde as famílias vivem e as relações que estabelecem com as instituições, com a alimentação e com o espaço social faz-se necessário para o entendimento do trabalho a ser realizado.

\section{CONSIDERAÇÕES FINAIS}

Com a realização do projeto de Acolhimento em Alimentação e Nutrição, o Projeto Creches Saudáveis buscou, através da educação popular, criar um elo entre as necessidades e expectativas da comunidade e a unidade de saúde, estabelecendo um vínculo duradouro entre a unidade educativa, as famílias da comunidade e a unidade de saúde.

No decorrer do trabalho, percebeu-se a importância de atividades como a territorialização e a antropometria, pois foram responsáveis por criar uma intersetorialidade entre os educadores, os nutricionistas e a comunidade, tornando o projeto conhecedor das características da comunidade e permitindo aos professores a utilização do alimento como elemento pedagógico.

Diante dos diagnósticos estabelecidos, pôde-se determinar a tendência de os escolares apresentarem peso elevado e pouca ocorrência de baixo peso em relação ao esperado, o que ressalta a importância de esses resultados serem levados em consideração na criação de novas políticas de saúde pública nessas regiões.

Tendo em vista o número de faltas nos encontros individuais do acolhimento, surgiu a proposta de realizá-lo de forma concomitante com os encontros coletivos, aumentando o vínculo entre as famílias e os nutricionistas, tendo em vista que os encontros seriam mais frequentes. Outra possibilidade seria substituí-lo por um atendimento único do nutricionista da Unidade de Saúde, proporcionando ações conjuntas e promovendo maior intersetorialidade. 


\section{REFERÊNCIAS}

BRASIL, Ministério da Saúde. Curvas de Crescimento da Organização Mundial da Saúde - OMS. 2002. Disponível em:

$<$ http://nutricao.saude.gov.br/sisvan.php?conteudo=curvas_cresc_oms $>$. Acesso em: $10 \mathrm{de}$ mar. 2010.

Disponível em:

. Portal da Saúde. O que é HumanizaSUS. Brasília: Ministério da Saúde, 2010.

$\leq$ http://portal.saude.gov.br/portal/saude/cidadao/visualizar_texto.cfm?Idtxt=28288 $>$. Acesso em: 07 de jun. de 2010.

. Secretaria de Atenção à Saúde. Coordenação-Geral da Política de Alimentação e Nutrição. Guia alimentar para a população brasileira: promovendo a alimentação saudável. Brasília: Ministério da Saúde, 2006.

Secretaria de Gestão Estratégica e Participativa. Departamento de Apoio à Gestão Participativa. Caderno de educação popular e saúde. Brasília: Ministério da Saúde, 2007.

BURITY, Valéria et al, M.F. Direito Humano à Alimentação Adequada no contexto da segurança alimentar e nutricional. Brasília, DF: ABRANDH, 2010. 204p.

CECANE/SC - Subcoordenação de Ensino e Extensão. Guia Creches Saudáveis.

Florianópolis, 2009 (mimeo.).

LOHMAN, Timothy; ROCHE Alexander F; MARTORELL, Reynaldo. Anthropometric standardization reference manual. Champaign, Illinois: Human Kinetics Books, 1991.

ONU. Assembléia Geral da Organização das Nações Unidas. Declaração Universal dos Direitos Humanos. Nova York, 1948. Disponível em:

$<$ http://www.onu-brasil.org.br/documentos_direitoshumanos.php $>$. Acesso em: 30 de mar. 2010.

WORLD HEALTH ORGANIZATION (WHO). Growth reference data for 5-19 years, 2007. Disponível em:<www.who.int/growthref/en/> . Acesso em: 30 de mar. 2010.

The WHO child growth standards, 2006. Disponível em:

$<$ http://www.who.int/childgrowth/standards/en/>. Acesso em: 30 de mar. 2010. 